\title{
TALLER I: ENFOQUES TEÓRICO-METODOLÓGICOS PARA EL ESTUDIO DE CERÁMICAS ARQUEOLÓGICAS
}

\section{LIBRO DE RESÚMENES}

18 de noviembre de 2016, Museo de La Plata

\section{ORGANIZADO POR}

\section{División Arqueología, Museo de la Plata}

Facultad de Ciencias Naturales y Museo, Universidad Nacional de La Plata

Dra. María Fabiana Bugliani

Dra. Violeta Di Prado

Dra. María Emilia Iucci
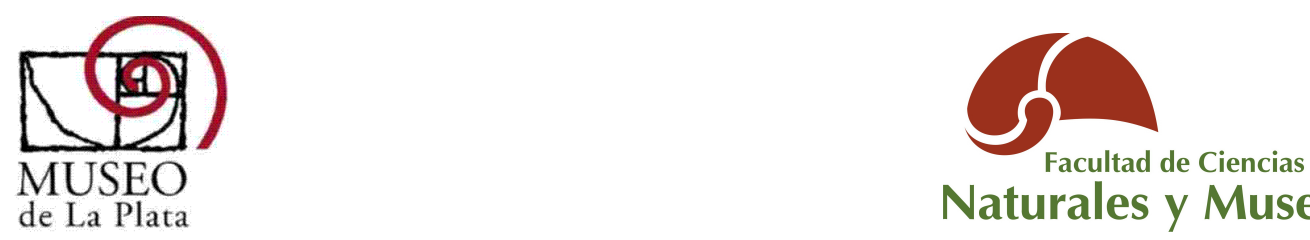
Naturales y Museo 


\section{Prólogo}

Desde los comienzos de la Arqueología argentina como disciplina científica y a través de diferentes enfoques y marcos conceptuales, se le ha asignado a la alfarería la responsabilidad de contribuir al abordaje y resolución de los más diversos problemas del pasado humano. Esta situación se debe, en parte, a que es uno de los materiales más abundantes y bien preservados en distintos tipos de contextos arqueológicos. Diferentes atributos de la cerámica han sido utilizados para identificar procesos de aprendizaje, disminución de la movilidad, intercambio, cambio cultural y diferenciación étnica, entre otros. Posiblemente, la alfarería "tenga algo que decir" sobre cada uno de estos temas, pero el grado de profundidad de sus aportes está en estrecha relación con la cantidad, variabilidad, preservación e integridad de la muestra bajo estudio, las condiciones de hallazgo y registro, la información contextual y cronológica disponible y las técnicas analíticas aplicadas.

Asimismo, las trayectorias de investigación sobre cerámicas arqueológicas en las diferentes regiones de Argentina presentan particularidades relacionadas con la profundidad y sistematicidad de los análisis, las estrategias teórico-metodológicas aplicadas, los objetivos buscados y los registros arqueológicos obtenidos. Las razones que explican este estado de la cuestión son complejas y entrelazan factores históricos, disciplinares, teórico-metodológicos, además de diferencias cuali-cuantitativas propias de la alfarería de cada una de las regiones. De modo general, los estudios ceramológicos poseen mayor profundidad temporal y refinamiento analítico en la región del NOA, aunque se han hecho considerables esfuerzos por avanzar en las investigaciones sobre cerámica en las restantes regiones del país.

Es precisamente la reflexión sobre estas cuestiones el disparador principal del taller. Aprovechando la cantidad y diversidad de equipos de trabajo de la Facultad de Ciencias Naturales y Museo de la UNLP que contemplan el estudio de la cerámica arqueológica entre sus metas, buscamos intercambiar experiencias, discutir criterios y posibilitar la resolución de inquietudes compartidas, con un énfasis en los marcos teórico-metodológicos desde los que se ha trabajado la alfarería, con especial foco en la definición de criterios, técnicas y metodologías aplicados a diferentes preguntas arqueológicas y antropológicas.

Comité Organizador

La Plata, 18 de Noviembre de 2016 


\title{
Forma, función y uso de la cerámica Guaraní
}

\author{
R. Costa Angrizani ${ }^{1,2}$ y M. L. Maravilla ${ }^{2}$ \\ ${ }^{1}$ CONICET-Centro de Investigaciones Científicas y Transferencia de Tecnología a la Producción. Matteri y España, \\ (3105) Diamante. E-mail: rcangrizani@gmail.com \\ ${ }^{2}$ Facultad de Ciencias Naturales y Museo, Universidad Nacional de La Plata. Av. 60 y 122, (1900) La Plata. \\ E-mail: marialauramaravilla@yahoo.com.ar
}

La forma de las vasijas cerámicas, además de servir como elemento distintivo para la definición de entidades arqueológicas, es el principal indicador adoptado para estimar la funcionalidad de los recipientes. En la arqueología de la Cuenca del Plata, desde el siglo XIX, se relaciona un estilo cerámico particular con la presencia del grupo etno-lingüístico guaraní en la región. Además de servir como marcador para la identificación de la Tradición Arqueológica Guaraní, desde la década de 1980, los elementos morfológicos de la alfarería pasaron también a ser tomados como atributos determinantes para la asignación de función. A partir del empleo de analogías entre los materiales y la información brindada en documentos etnohistóricos, se estableció una taxonomía para clasificar las vasijas según su funcionalidad primaria. El empleo de tal razonamiento permitió ordenar la variabilidad interna de los conjuntos arqueológicos y clasificarlos de acuerdo a categorías etnográficas. A pesar de esto, el uso irreflexivo del modelo incidió en interpretaciones mecanicistas. Enfocados en esta problemática, en esta exposición presentamos las investigaciones sobre la alfarería guaraní de sitios en la cuenca del río Uruguay. Se discuten las posibilidades y los límites del modelo etnohistórico de forma-función, y se contrastan las interpretaciones con los resultados de análisis arqueométricos (ácidos grasos e identificación de microrrestos paleobotánicos).

Palabras clave: Tradición arqueológica Guaraní, Tecnología alfarera, Analogía etnohistórica, Arqueometría, Tierras Bajas Sudamericanas. 


\title{
Abordaje de la historia de vida de la alfarería arqueológica del Delta Superior del río Paraná (sur de Entre Ríos)
}

\author{
V. Di Prado ${ }^{1}$, C. Castroo $^{1}$ y C. Silva ${ }^{1}$ \\ ${ }^{1}$ CONICET - División Arqueología, Facultad de Ciencias Naturales y Museo, Universidad Nacional de La Plata. Museo de La Plata, \\ Paseo del bosque s/n (1900), La Plata. E-mails: violetadiprado@hotmail.com, canelamdq@hotmail.com, karitosilva@gmail.com
}

A pesar de la abundancia y diversidad de la alfarería del Delta Superior del río Paraná y de su relevancia para la arqueología del área, hasta muy recientemente los análisis estuvieron centrados solo en sus aspectos formales y decorativos. En esta contribución se presentan las estrategias metodológicas implementadas para abordar la cerámica del área, que incluyen análisis de atributos a nivel macroscópico, sobre la base de los cuales se definen unidades de análisis y criterios de muestro, para la posterior aplicación de técnicas arqueométricas (e.g., petrografía de pastas, difracción de rayos $\mathrm{X}$, análisis radiográfico, análisis térmico diferencial y termogravimétrico). El primer paso fundamental consiste en reconstruir las piezas mediante remontajes y en estimar el número mínimo de vasijas (NMV). La caracterización de las etapas de producción permite identificar diversos modos de hacer, que integrados con información contextual y cronológica posibilitan discutir procesos de cambio y continuidad en las tradiciones de manufactura, y de diferenciación e interacción social entre los grupos locales y los de áreas vecinas. El uso de la cerámica en contextos domésticos y funerarios, y como material constructivo es abordado mediante el relevamiento de huellas generadas por uso, la estimación de propiedades funcionales derivadas de la forma y el tamaño de las vasijas, y el análisis microscópico de microrrestos biosilíceos y almidones en las adherencias. Por último, se registran huellas de alteración no generadas por uso para inferir los procesos y agentes posdepositacionales, tanto culturales como naturales, que afectaron los contextos.

Palabras Clave: Delta Superior del rio Paraná, Alfarería, Prácticas de producción y uso, Análisis a nivel macroscópico, Técnicas arqueométricas. 


\title{
Relatos arqueológicos y tiestos: lo que es posible decir de la dinámica de vida en los sitios EI Shincal de Quimivil, Los Colorados y Quillay a través de los fragmentos de cerámica
}

\author{
M. Giovannetti ${ }^{1,2}$, G. Cochero ${ }^{1}$, J. Spina ${ }^{1,2}$, E. Ferraris ${ }^{1}$ e I. Fasciglione ${ }^{1}$ \\ ${ }^{1}$ División Arqueología, Facultad de Ciencias Naturales y Museo, Universidad Nacional de La Plata. Museo de La Plata, Paseo del \\ Bosque s/n (1900), La Plata. E-mails: mgiovannetti@conicet.gov.ar, gcochero@gmail.com, josefinaspina@gmail.com, \\ edgardo_ferraris@hotmail.com,ivan fachi@hotmail.com \\ ${ }^{2}$ CONICET
}

Recuperados a partir de excavaciones sistemáticas, recolecciones de superficie y reestudios de colecciones antiguas depositadas en museos, hemos estudiado cientos de fragmentos de cerámica correspondientes a tres sitios arqueológicos de la provincia de Catamarca: El Shincal de Quimivil, Los Colorados y Quillay. Los proyectos de investigación están centrados en el período inkaico aunque reconociendo que en Los Colorados existe ocupación multicomponente llegando hasta los momentos del formativo de la región. En todos los casos se conformaron conjuntos de tiestos separados correspondientemente por su contexto, buscando al mismo tiempo a partir del remontaje o la identificación de familias de tiestos obtener una magnitud más precisa de la cantidad de piezas representadas. Los fragmentos se analizan luego a partir de un esquema de variables que buscan regularidad en la descripción de las características propias tales como tratamientos de las superficies, tipos de cocción, medidas, estados de desgaste, fracturas, decoración, entre otras, que permiten una identificación morfológica estilística de diferente grado de precisión (dependiente de la información posible de registrar en cada caso). La información obtenida se analiza estadísticamente para tener una noción sobre las tendencias en relación a las variables usadas. Estos análisis han permitido vincular la evidencia cerámica con diferentes tópicos de nuestras investigaciones como por ejemplo prácticas vinculadas a la producción metalúrgia (sitio Quillay), cronología relativa y materialidad en los campos de cultivo (Los Colorados), producción de alimentos y consumo en festividades masivas, ritualidad y comensalismo (El Shincal de Quimivil).

Palabras clave: Fragmentos cerámicos, Catamarca, Inka 


\title{
Los estudios de la cerámica arqueológica del Valle de Hualfín (Belén, Catamarca): líneas metodológicas para el abordaje de problemáticas alfareras en los paisajes prehispánicos
}

\author{
M. E. Iucci ${ }^{1,2}$, B. Balesta ${ }^{1}$, F. Wynveldt ${ }^{1,2}$ y N. Zagorodny ${ }^{1}$ \\ ${ }^{1}$ Laboratorio de Análisis Cerámico, Facultad de Ciencias Naturales y Museo, Universidad Nacional de La Plata. Calle 64 Nº 3 Lab. \\ 18, (1900) La Plata. E-mails: emiliaiucci@yahoo.com.ar, barbarabalesta@hotmail.com,wynveldtf@fcnym.unlp.edu.ar, \\ nzagorodny@hotmail.com \\ ${ }^{2}$ CONICET
}

Los estudios cerámicos desarrollados por el equipo de trabajo del LAC de la FCNyM en el Valle de Hualfín (depto de Belén, prov. de Catamarca) se han centrado en el conjunto de las alfarerías que fueron elaboradas en el valle, o que llegaron de otras regiones, durante el primer milenio de la Era hasta mediados del segundo. Estos estudios se llevaron a cabo en base a un enfoque relacional, que considera a la cerámica como un participante más de las prácticas sociales que contribuye a configurar los paisajes locales en sus distintas dimensiones. Este enfoque ha permitido situar a la cerámica en las distintas esferas de la vida de los antiguos habitantes, en las coyunturas sociopolíticas y en las dinámicas de interacción intra e interregionales.

En este contexto, las líneas de investigación se desarrollaron en base a interrogantes tales como cuáles son las causas que explican la variabilidad de los conjuntos, cómo se organizaba la producción cerámica, en qué prácticas participaba y cómo las conceptualizaciones cognitivas estaban involucradas en la producción alfarera. Estas problemáticas han sido abordadas tanto a través de métodos tradicionales para el tratamiento de material cerámico, como por medio de la aplicación de técnicas arqueométricas y de semiótica de las imágenes materiales visuales sobre conjuntos alfareros de diferentes contextos (funerarios, domésticos, de producción) y origen (de excavación y colecciones con distinto grado de documentación).

Palabras clave: Valle de Hualfin, Tradiciones alfareras, Estudios del paisaje, Estudios cerámicos, Arqueometría cerámica 


\title{
Variabilidad cerámica presente en los caminos de la región de Londres de Quimivil
}

\author{
R. A. Moralejo ${ }^{1,2}$, M. Aventín Moretti ${ }^{1}$ \\ ${ }^{1}$ División Arqueología, Facultad de Ciencias Naturales y Museo, Universidad Nacional de La Plata, Museo de La Plata, Paseo del \\ Bosque s/n (1900) La Plata. E-mails: reinaldomoralejo@yahoo.com.ar, milagrosaventin@hotmail.com \\ ${ }^{2}$ CONICET
}

Para los Inkas, la idea de "camino" constituyó un complejo sistema infraestructural compuesto por distintos hitos culturales que respondían a las diferentes necesidades del imperio tanto administrativas como de transporte, comunicación y hasta limítrofes. Si bien existen diferentes investigaciones al sur del Valle de Hualfín y en el interfluvio de la Sierra de Zapata, son pocos los estudios sobre la alfarería presente a lo largo de los caminos. Al ser la cerámica un instrumento diagnóstico que permite la determinación de los cambios ocurridos en el tiempo y en el espacio de las sociedades del pasado, y considerando que los fragmentos cerámicos pueden ser interpretados como una parte del registro material, se planteó como objetivo principal la caracterización de los estilos cerámicos presentes a lo largo de los caminos recorridos, así como también aquellos encontrados en los sitios asociados. El análisis consistió en observaciones macroscópicas orientadas a dilucidar aspectos sobre la decoración, morfología y tecnología lo cual permitió realizar aproximaciones crono-culturales relacionadas con los diferentes momentos de utilización de las vías en la región. Estos trabajos conducen a pensar que la presencia de determinados objetos materiales estaría señalando diferentes prácticas cotidianas vinculadas con el rol que estas redes viales tenían dentro del paisaje resignificado por los Inkas.

Palabras Clave: Caminos longitudinales y transversales, El Shincal de Quimivil, Catamarca, Noroeste argentino 


\title{
Tecnología cerámica y prácticas cotidianas en grupos arqueológicos de la Cuenca del Plata
}

\author{
M. C. Paleo ${ }^{1}$, M. Pérez Meroni ${ }^{1}$, N. Ghiani Echenique ${ }^{1}$, G. Acuña ${ }^{1}$, P. León ${ }^{1}$ y M. Auge $^{1}$ \\ ${ }^{1}$ Laboratorio de Análisis Cerámico, Facultad de Ciencias Naturales y Museo, Universidad Nacional de La Plata. Calle 64 N 3 Lab. \\ 18, (1900) La Plata. E-mails: mcpaleo@fcnym.unlp.edu.ar, mperezmeroni@yahoo.com.ar, naiqueng@gmail.com, \\ holodorin_gri@hotmail.com,paulaleon1@hotmail.com,meli_aug47@hotmail.com
}

Se presentan los estudios sobre tecnología cerámica realizados sobre material proveniente de grupos cazadores, recolectores y pescadores del Holoceno tardío, que habitaron el litoral fluvial bonaerense de los actuales partidos de Magdalena y Punta Indio, (Buenos Aires) hace aproximadamente 2000 años. Uno de los objetivos propuestos en las investigaciones fue indagar acerca de las prácticas cotidianas de estos grupos en relación al procesamiento y consumo de diferentes recursos. De este modo, se han utilizado diferentes metodologías y líneas de evidencia e implementado estudios analíticos cuya lectura conjunta permite realizar interpretaciones en torno a la tecnología cerámica y a las prácticas asociadas. Se han realizado caracterizaciones morfo-funcionales, reconstrucciones en $3 \mathrm{D}$ de los contenedores, aplicado técnicas arqueométricas para el análisis de pasta, análisis de residuos orgánicos y de microrrestos vegetales. Los resultados obtenidos han sido interpretados en forma conjunta con la información procedente del registro arqueofaunístico, arquebotánico y cronológico. Se han caracterizado estilos tecnológicos en los conjuntos analizados y prácticas de procesamiento de distintos recursos. Asimismo, en los últimos años se desarrolla una línea de trabajo que aborda cerámicas históricas, específicamente pipas de caolín de sitios rurales del siglo XIX, cuyo análisis aporta a interpretar las prácticas asociadas al consumo de fumitorios en momentos históricos.

Palabras clave: Tecnología cerámica, Prácticas cotidianas, Grupos arqueológicos, Estudios arqueométricos 


\title{
Una aproximación metodológica integrativa a la cerámica arqueológica del valle del Cajón
}

\author{
M. C. Scattolin ${ }^{1,2}$, M. F. Bugliani ${ }^{1,2}$ y L. Pereyra Domingorena ${ }^{2}$ \\ ${ }^{1}$ Facultad de Ciencias Naturales y Museo, Universidad Nacional de La Plata. \\ ${ }^{2}$ CONICET- IDECU-Museo Etnográfico-UBA, Moreno 350 (1091) CABA. \\ E-mails: cscattolin@gmail.com, fbugliani@gmail.com, lucasdomingorena@gmail.com
}

En esta presentación buscamos dar a conocer el programa de investigación, con diferentes vías de análisis, que estamos implementando para el estudio de la cerámica del valle del Cajón (Dpto. Santa María, Catamarca). Se detallan los análisis petrográficos, morfo-funcionales y estilísticos, efectuados a fragmentos y recipientes cerámicos reconstruidos para los sitios Cardonal, Bordo Marcial, Yutopián y Loma l'Ántigo. Por un lado, se han efectuado estudios de tecnología cerámica, a través del examen petrográfico de las pastas y del análisis químico de arcillas recolectadas en el área de estudio. A la vez, hemos desarrollado una metodología para la reconstrucción de formas de vasijas a fin de recopilar un repertorio morfológico lo más completo posible. Por otro lado, hemos identificado los recursos compositivos, no sólo determinando los elementos del diseño aislados sino rastreando las maneras en que estos elementos se articulan y se despliegan en el espacio de representación. Dado que la unidad de análisis considerada es la vasija, integramos estas distintas vías de estudio en cada una de las reconstrucciones y conjuntos de fragmentos identificados. A modo de síntesis, se presenta una caracterización de las producciones cerámicas existentes desde el Período Formativo hasta el Período de Desarrollos Regionales.

Palabras clave: Valle del Cajón, Pastas cerámicas, Recursos iconográficos, Repertorios de forma 


\title{
La alfarería del valle Calchaquí (Salta): especificidad, movimientos y usos en una perspectiva de larga duración
}

\author{
M. Sprovieri ${ }^{1,4}$, S. Barbich ${ }^{1,4}$, S. Cohen ${ }^{2}$ y A. P. Cevidanes ${ }^{3}$ \\ ${ }^{1}$ División Arqueología, Facultad de Ciencias Naturales y Museo, Universidad Nacional de La Plata. Anexo Museo, Av. 122 y 60 \\ (1900) La Plata. E-mails: msprovieri@fcnym.unp.edu.ar, sbarbich@fcnym.unlp.edu.ar \\ 2 Área Etnografía, Museo Etnográfico “J. B. Ambrosetti”, Facultad de Filosofía y Letras, UBA. Moreno 350 (1090) CABA. \\ E-mail: sebafcohen@hotmail.com \\ ${ }^{3}$ Facultad de Filosofía y Letras, UBA. E-mail: anapaulacevidanes@gmail.com \\ ${ }^{4}$ CONICET, Puan 480 (1406) CABA.
}

La alfarería tardía del valle Calchaquí, Provincia de Salta, ha sido tradicionalmente incluida dentro del amplio espectro de la cerámica "santamariana", la cual fue definida a partir de materiales del valle de Yocavil. Es por ello que consideramos que la primera aún requiere de una profundización en su análisis y una caracterización propia. En este sentido, hemos avanzado en la distinción de variantes alfareras en nuestra región de estudio (valle Calchaquí medio), así como en los procesos de circulación y consumo de vasijas tanto en esferas domésticas como funerarias. Estos aspectos se han abordado a partir de un enfoque estilístico que combina análisis de materiales de colecciones de museo y de excavaciones propias. Además, recientemente, hemos iniciado aproximaciones a los procesos de producción cerámica, sobre los que esperamos profundizar. Estas líneas de análisis se abordan desde un enfoque que busca estudiar la materialidad calchaquí en una perspectiva de larga duración, integrando información arqueológica y etnográfica. A partir de esto se pretende aportar a la construcción de un marco culturalmente sensitivo sobre las maneras en que distintas materialidades son incorporadas a diversas esferas de la práctica social, sus relaciones contextuales y usos, y posiblemente sus valoraciones.

Palabras clave: Cerámica, Valle Calchaqui, Especificidad regional, Arqueología, Etnografía 


\title{
La cerámica en la Meseta de Somuncurá (provincia de Río Negro): perspectivas de análisis
}

\author{
J. Vargas Gariglio ${ }^{1}$, E. Terranova ${ }^{1,2}$ y D. Hermo ${ }^{1,2}$ \\ ${ }^{1}$ División Arqueología, Museo de La Plata, Facultad de Ciencias Naturales y Museo, Universidad Nacional de La Plata. Museo de \\ La Plata, Paseo del Bosque s/n (1900)La Plata. E-mails: lacavanajuja@yahoo.com.ar, quiqueterra@gmail.com, \\ dhermo@unlp.edu.ar \\ ${ }^{2}$ CONICET
}

Los estudios arqueológicos sistemáticos en la porción rionegrina de la meseta de Somuncurá, comenzados en 2002, nos han permitido avanzar en el conocimiento de las sociedades cazadoras-recolectoras que la poblaron. A través de diferentes proyectos hemos abordado problemáticas como la tecnología lítica y la estructura de recursos líticos, el arte rupestre, así como también comenzamos estudios zooarqueológicos y tafonómicos y realizamos los primeros anclajes cronológicos. Uno de los desafíos más recientes fue el toparnos con un tipo de evidencia con el que no habíamos dialogado mucho: la cerámica. La Meseta de Somuncurá es un extenso macizo volcánico ubicado entre las provincias de Chubut y Río Negro, con altitudes superiores a $1000 \mathrm{msnm}$. Como resultado de distintos procesos erosivos, en la meseta se registran bajos de distintas dimensiones. Entre ellos se encuentra la Laguna Azul. Los trabajos de excavaciones y prospecciones realizados en 2014 permitieron registrar una muestra de tecnología cerámica. Los fechados obtenidos a partir de las excavaciones ubican este tipo de tecnología entre $1906 \pm 26$ y $1756 \pm 28$ años AP. El objetivo de esta participación es exponer el caso de estudio y presentar las líneas de análisis, que hasta el momento son: 1) análisis petrográfico de pastas y 2) análisis de difracción de rayos X. Dependiendo de las características de la muestra y de los aportes de los participantes del taller se evaluará aplicar otras técnicas como: activación neutrónica (NAA), análisis de sustancias lipídicas, microscopía electrónica de barrido y microsonda acoplada (SEM + EDAX).

Palabras clave: Norpatagonia, Cazadores-recolectores, Cerámica 